\title{
Study of Non-Standard Charged-Current Interactions at the MOMENT experiment
}

\author{
Jian Tang*, Yibing Zhang \\ School of Physics, Sun Yat-Sen University, 510275, Guangzhou, China \\ E-mail: tangjian5@mail.sysu.edu.cn
}

\begin{abstract}
MuOn-decay MEdium baseline NeuTrino beam experiment (MOMENT) is a next-generation accelerator neutrino experiment, which can be used to probe new physics beyond Standard Model. We try to simulate neutrino oscillations confronting with Charged-Current and Non-Standard neutrino Interactions(CC-NSIs) at MOMENT. These NSIs could alter neutrino production and detection processes and interfere with neutrino oscillation channels. We separate a perturbative discussion of oscillation channels at near and far detectors, and analyze parameter correlations with the impact of CC-NSIs. Taking $\delta_{c p}$ and $\theta_{23}$ as an example, we find that CC-NSIs can induce bias in precision measurements of standard oscillation parameters. In addition, a combination of near and far detectors using Gd-doped water cherenkov technology at MOMENT is able to provide good constraints of CC-NSIs happening to the neutrino production and detection processes.
\end{abstract}

The 19th International Workshop on Neutrinos from Accelerators-NUFACT2017

25-30 September, 2017

Uppsala University, Uppsala, Sweden

\footnotetext{
* Speaker.
} 


\section{Introduction}

In the past decades, we have seen enormous progress from neutrino oscillation experiments using solar, atmospheric, accelerator and reactor neutrinos [1, 2, 3, 4, 5, 6, 7, 8]. According to a global analysis of these neutrino oscillation experiments [9, 10,11, 12], mixing angles $\theta_{12}, \theta_{13}$ $\& \theta_{23}$ and mass square differences $\Delta m_{21}^{2} \&\left|\Delta m_{31}^{2}\right|$ have so far been well measured. However, a deviation from $\theta_{23}=45^{\circ}$ causes an octant degeneracy problem in certain neutrino oscillation channels $[13,14]$. Nonetheless, the Dirac CP phase describing the difference between matter and anti-matter as well as the sign of $\Delta m_{31}^{2}$ have not been well constrained yet. Though recent results from T2K [15] and NOvA [16] disfavor the inverted mass hierarchy at a low confidence level and give hints of $\delta_{C P} \approx-90^{\circ}$, we expect more data to draw a solid conclusion or further call for the next-generation experiments such as accelerator neutrino oscillation experiments like DUNE [17] and T2HK [18], the medium-baseline reactor experiments [19] like JUNO [20] and RENO-50 [21], atmospheric neutrino experiments like INO [22], PINGU [23] and KM3Net [24].

With more particle contents in new physics models, it might contain the sub-leading effects induced by non-standard neutrino interactions (NSIs) in nature. Effective operators have been adopted to link neutrino mass models and NSIs. A review of NSIs is given in detail in Ref. [25, 26]. With the help of an effective field theory, we can generally integrate out the mediator/propagator in the Feynman diagram and keep four fermions contact with each other. New physics scale is then embedded into the effective coupling constant $\varepsilon_{\alpha^{\prime} \beta^{\prime}}^{\alpha \beta}$ where $\alpha / \beta$ or $\alpha^{\prime} \beta^{\prime}$ are the related fermion flavours. We have reached an era of precision measurements of neutrino mixing parameters after an establishment of neutrino oscillation. It is promising for us to develop better neutrino detectors to search for sub-leading NSIs in the current and next-generation neutrino oscillation experiments as a complementary to the new physics search with the high intensity machine at the collider.

The MuOn-decay MEdium baseline NeuTrino beam experiment (MOMENT) is a next-generation accelerator neutrino experiment proposed for discovery of leptonic CP violation [27]. The atmospheric neutrino flux is a serious hindrance. A new detector technology, however, might overcome the barrier and lead to a discovery of the $\mathrm{CP}$ violating phase in the framework of 3-flavour neutrino oscillations [28], which complement the study at T2K and NOvA. In addition, a comprehensive study of the bounds on NSI parameters has been carried out [29]. With the help of a perturbation theory, neutrino oscillation probabilities in the presence of source/detector and matter NSIs can be found in the reference [30], which is motivating further study and optimization of new experimental proposals to pin down the current bounds. The first glimpse of NSI effects during neutrino propagation in matter at MOMENT has been shown in the reference [31]. However, the impact of source and detector NSIs associated with charged-current interactions has not been discussed. It is necessary to bring source/detector NSIs for a complete analysis at MOMENT where neutrinos are produced by muon decays. We explore the charged current NSIs effects at MOMENT, focusing on the precision measurement of standard neutrino mixing parameters and constraints of NSI parameters.

\section{Comments on neutrino oscillation channels}

The formalism of NSI is a general way of studying the impacts of new physics in neutrino 
oscillations. The CC-NSIs imposed on the production and detection are two different types: the NSIs involved in the muon decay production process are related to charged leptons, while the NSIs involved in the detection process are associated with quarks. For simplicity, we have restricted the operators to $(V-A)(V-A)$ structure while other Lorentz structures are either helicity suppressed or very small due to their contributions by higher order corrections. The NSIs at the detector are parametrized by $\varepsilon_{\alpha \beta}^{d}$ which give the strength of NSIs relative to $G_{F}$. Similarly, we factorize the CC-NSIs in the muon decays with $\varepsilon_{\gamma \delta}^{s}$. Thus, the oscillation probability is given by:

$$
P\left(v_{\delta}^{s} \rightarrow v_{\beta}^{d}\right)=\left|\left[\left(1+\varepsilon^{d}\right)^{T} e^{-i \mathscr{H} L}\left(1+\varepsilon^{s}\right)^{T}\right]_{\beta \delta}\right|^{2}
$$

Here the Hamiltonian takes the form of $\mathscr{H}=U \operatorname{diag}\left(E+\frac{m_{1}^{2}}{2 E}, E+\frac{m_{2}^{2}}{2 E}, E+\frac{m_{3}^{2}}{2 E}\right) U^{\dagger}$ and $\mathrm{U}$ is the PMNS mixing matrix relating the neutrino flavour eigenstates to mass eigenstates $\left|v_{\alpha}\right\rangle=\sum_{i} U_{\alpha i}^{*}\left|v_{i}\right\rangle$. The $\varepsilon^{s}$ and $\varepsilon^{d}$ are the charged-current NSI matrices for the production and detection, respectively. There are 18 NSI real parameters in total because each complex element $\varepsilon_{\alpha \beta}^{s / d}$ consists of the amplitude $\left|\varepsilon_{\alpha \beta}^{s / d}\right|$ and the phase $\phi_{\alpha \beta}^{s / d}$. Since near and far detectors will be used in the simulation later, we will discuss the oscillation channels at a short and far distance separately.

\subsection{Oscillation channels at a near detector}

Here a near detector means detecting neutrinos at a distance of $\mathrm{O}(100)$ meters. In the standard oscillation frame without non-standard interactions, $v_{\mu}\left(\bar{v}_{\mu}\right)$ and $v_{e}\left(\bar{v}_{e}\right)$ can not develop neutrino oscillation patterns in such a short distance and their probabilities are equal to 1 . However, NSIs are able to generate zero-distance effects. After dropping the terms $O\left(\varepsilon^{2}\right)$, we approximate the probabilities as Eq. (2.2) and Eq. (2.3):

$$
\begin{gathered}
P_{v_{e}^{s} \rightarrow v_{e}^{d}}^{N D}\left(P_{\bar{v}_{e}^{s} \rightarrow \bar{v}_{e}^{d}}^{N D}\right) \approx 1+2\left|\varepsilon_{e e}^{s}\right| \cos \phi_{e e}^{s}+2\left|\varepsilon_{e e}^{d}\right| \cos \phi_{e e}^{d} \\
P_{v_{\mu}^{s} \rightarrow v_{\mu}^{d}}^{N D}\left(P_{\bar{v}_{\mu}^{s} \rightarrow \bar{v}_{\mu}^{d}}^{N D}\right) \approx 1+2\left|\varepsilon_{\mu \mu}^{s}\right| \cos \phi_{\mu \mu}^{s}+2\left|\varepsilon_{\mu \mu}^{d}\right| \cos \phi_{\mu \mu}^{d}
\end{gathered}
$$

It is easy to see that $P\left(\bar{v}_{e}^{s} \rightarrow \bar{v}_{e}^{d}\right) / P\left(\bar{v}_{\mu}^{s} \rightarrow \bar{v}_{\mu}^{d}\right)$ deviates from unity with some constant terms in the presence of relevant NSI parameters $\varepsilon_{e e}^{s}$ and $\varepsilon_{e e}^{d}\left(\varepsilon_{\mu \mu}^{s}\right.$ and $\left.\varepsilon_{\mu \mu}^{d}\right)$. If neutrinos are produced with charged lepton decays and detected by identifying the same charged leptons, the contribution of $\varepsilon_{e e}^{s}$ to the probability is equivalent to $\varepsilon_{e e}^{d}$ and then the sensitivity to these two parameters should be the same at the near detector. It is a discovery of new physics to observe zero-distance effects at near detectors for disappearance or appearance channels.

\subsection{Oscillation channels at a far detector}

Oscillation patterns get more complicated as soon as we consider channels suitable for the far detector at MOMENT. In the standard framework describing three neutrino mixings, the probability of $v_{\mu} \rightarrow v_{e}$ channel is calculated by a simple change of sign of the $\sin \delta$ term in a T-reversed channel of $v_{e} \rightarrow v_{\mu}$. Due to CC-NSIs, $v_{\mu} \rightarrow v_{e}$ and $v_{e} \rightarrow v_{\mu}$ probabilities are not so obvious any more. We perturbatively derive the explicit expressions of their probabilities in vaccum, considering $\alpha=$ $\frac{\Delta m_{21}^{2}}{\Delta m_{31}^{2}} \approx 0.03, s_{13}=\sin \theta_{13} \approx 0.15$ and NSI parameters as small numbers. In order to clearly show the impacts of NSIs, we can split the $P_{v_{\mu} \rightarrow v_{e}}^{F D}\left(P_{v_{e} \rightarrow v_{\mu}}^{F D}\right)$ into a sum of three terms: the standard oscillation 

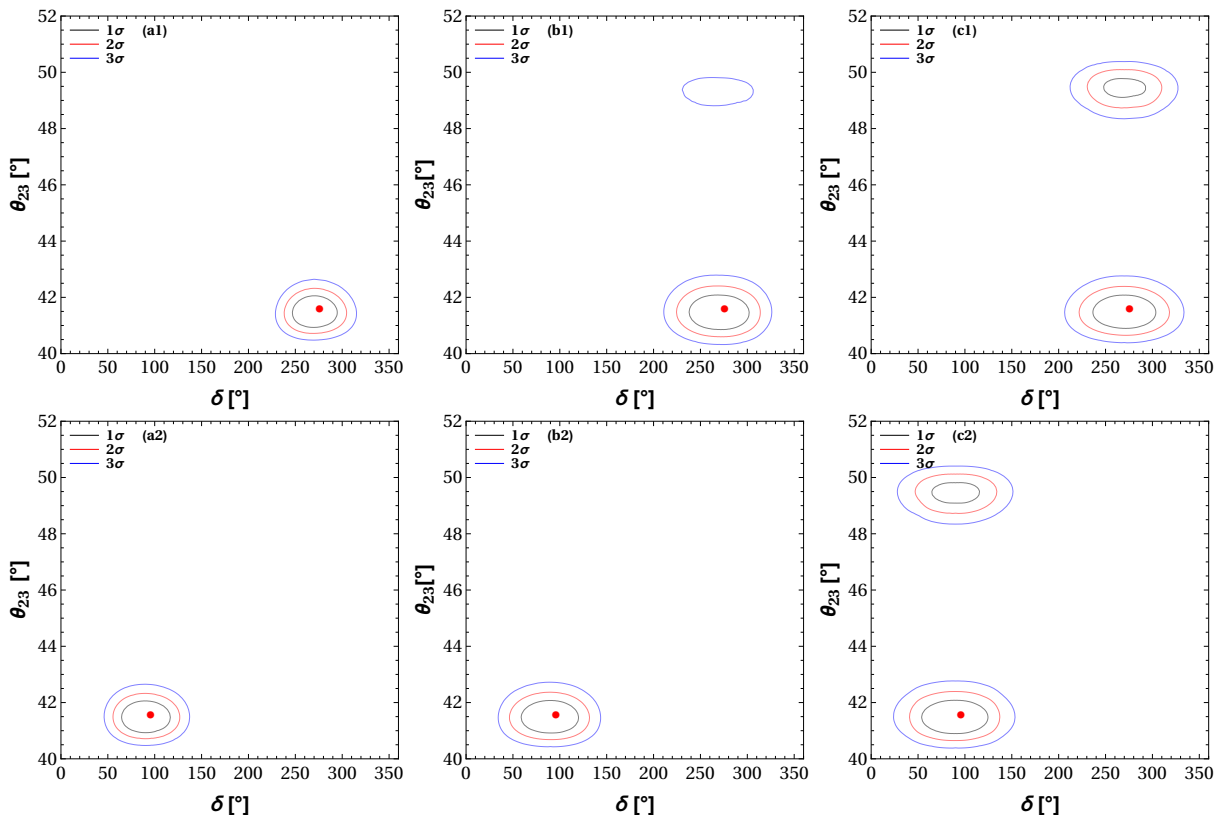

Figure 1: The allowed region for $\theta_{23}-\delta$ for MOMENT. Panel (a) shows the determination of $\delta$ and $\theta_{23}$ in the case of the standard three flavour frame. In panel (b) we only consider the NSIs: $\varepsilon_{e \mu}^{s}, \varepsilon_{e \tau}^{s}$ and $\varepsilon_{\tau e}^{d}$ which are related to $v_{\mu}$ appearance channels. (c) shows the effects of those NSIs related to the $v_{\mu}\left(\bar{v}_{\mu}\right)$ and $v_{e}\left(v_{e}\right)$ appearance channels: $\varepsilon_{e \mu}^{s}, \varepsilon_{e \mu}^{d}, \varepsilon_{e \tau}^{s}, \varepsilon_{\mu e}^{s}, \varepsilon_{\mu e}^{d}, \varepsilon_{\tau e}^{d}$ and marginalization ranges are within current bounds. All of the corresponding phases can vary in $(0,2 \pi)$. The red points in all panels indicate the true values.

term $P_{v_{\mu} \rightarrow v_{e}}^{S M}\left(P_{v_{e} \rightarrow v_{\mu}}^{S M}\right)$, the dominant order of $\mathscr{O}\left(\varepsilon s_{13}\right)$ NSI oscillatory term $P_{v_{\mu} \rightarrow v_{e}}^{N S I\left(\varepsilon s_{13}\right)}\left(P_{v_{e} \rightarrow v_{\mu}}^{N S I\left(\varepsilon_{13}\right)}\right)$ and the sub-dominant order of $\mathscr{O}(\alpha \varepsilon)$ NSI oscillatory term $P_{v_{\mu} \rightarrow v_{e}}^{N S I(\alpha \varepsilon)}\left(P_{v_{e} \rightarrow v_{\mu}}^{N S I(\alpha \varepsilon)}\right)$.

\section{Physics performance of MOMENT}

\subsection{Impacts on precision measurements of standard mixing parameters by CC-NSIs}

The CKM mixing matrix is well measured in the quark sector at the sub-percent level [32], while mixing parameters in the lepton sector are far away from such a precision. It is very likely for the next-generation experiment like MOMENT to achieve the goal of doing precision measurements. Fig. 1 demonstrates precision measurements with/without NSIs. Panels (a1) and (a2) show the determination of $\delta_{\mathrm{cp}}$ and $\theta_{23}$ in the case of the standard neutrino oscillation without NSIs. By running MOMENT, we can determine the mixing angle $\theta_{23}$ with an error bar of one degree at the $3 \sigma$ confidence level, while the precision for $\delta_{\mathrm{cp}}$ is good enough. In sub-figure (b) and (c), NSIs happening at the source and detector are turned on. All the corresponding CC-NSI phases can vary within $(0,2 \pi)$. As can be seen from panels (c1) and (c2), the degeneracy even shows up at high confidence levels when we consider all the relevant NSI parameters. We might get into the wrong best-fit region if we neglect the CC-NSIs from the new physics. A combination of different neutrino oscillation experiments might resolve such an ambiguity and finish the task of precision measurements of neutrino mixing parameters to the same level as quark mixing parameters. Or we need a more powerful machine, such as a neutrino factory. 


\begin{tabular}{|c|c|c|c|c|}
\hline Parameter & ND constraints & FD constraints & ND+FD constraints & Current bounds \\
\hline$\left|\varepsilon_{e e}^{s}\right|$ & 0.027 & 0.028 & 0.018 & 0.025 \\
\hline$\left|\varepsilon_{e \mu}^{s}\right|$ & 0.023 & 0.018 & 0.014 & 0.03 \\
\hline$\left|\varepsilon_{\mu e}^{s}\right|$ & 0.025 & 0.021 & 0.015 & 0.025 \\
\hline$\left|\varepsilon_{\mu \mu}^{s}\right|$ & 0.028 & 0.029 & 0.019 & 0.03 \\
\hline$\left|\varepsilon_{e e}^{d}\right|$ & 0.027 & 0.028 & 0.027 & 0.041 \\
\hline$\left|\varepsilon_{e \mu}^{d}\right|$ & 0.023 & 0.015 & 0.013 & 0.026 \\
\hline$\left|\varepsilon_{\mu e}^{d}\right|$ & 0.025 & 0.022 & 0.025 & 0.025 \\
\hline$\left|\varepsilon_{\mu \mu}^{d}\right|$ & 0.028 & 0.03 & 0.028 & 0.078 \\
\hline
\end{tabular}

Table 1: Expected 90\% credible regions on NSI parameters with a single detector or a combination of near and far detectors at the MOMENT experiment. Here NSI parameters are assumed to be real, or NSI-induced $\mathrm{CP}$ phases are switched off.

\subsection{Constraints of NSI parameters}

We have introduced the NSIs by integrating the potential heavy propagator from the new physics scale based on the effective theory. Each NSI parameter has a magnitude which tells us the strength of new couplings and its associated phase to bridge the CP violating story. In this section we discuss the constraints on source and detector NSIs from the far and near detector, respectively. For the former case, Table 1 demonstrates the sensitivity of MOMENT in constraining the NSI parameters using the single-parameter-fit at 90\% C.L. In a comparision of the current bounds and the expected limits from our simulation, we find that the MOMENT experiment with running time of 5+5 years has a potential to improve the constraints for the CC NSIs. The far detector has a good sensitivity to NSI parameters, especially for $\varepsilon_{e \mu}^{d}$ and $\varepsilon_{\mu e}^{s}$. Almost all NSI-induced phases change the exclusion limits severely except the e-mu sector. Therefore, MOMENT using muon-decay beams has its unique capability of improving the constraints on $\varepsilon_{e \mu}^{d}$ and $\varepsilon_{\mu e}^{s}$.

\section{Summary and conclusions}

The next-generation accelerator neutrino experiment MOMENT intends to produce the powerful neutrino beam with an energy of $\mathrm{O}(100) \mathrm{MeV}$ by muon decays and leaves plenty of room for detector selections and physics study. At this energy range, quasi-elastic neutrino interactions dominate the detection process and backgrounds from $\pi^{0}$ are highly suppressed. Compared with traditional superbeams from charged meson decays where intrinsic backgrounds have to be alleviated by the off-axis technology like T2K and NOvA, beams from muon decays are cleaner neutrino sources and good at a detection of new physics. CC-NSIs happening at neutrino productions and detections point to the new phenomenon, where a neutrino produced or detected together with the charged lepton will not necessarily share the same flavour, and flavour conversion is present already at the interaction level and "oscillations" can occur at zero distance. With the capability of flavour and charge identifications, we have an opportunity to use eight appearance and disappearance oscillation channels in the physics study. We have chosen the advanced neutrino detector using the Gd-doped Water Cherenkov technology and studied neutrino oscillations confronting with CC-NSIs at the MOMENT experiment. Our study has shown that a combination of near and 
far detectors at MOMENT is able to provide lower bounds on CC-NSIs where a factor of about two can be envisaged for most of parameters compared with the current experimental bounds.

\section{Acknowledgement}

This work is supported by the start-up funding from SYSU, the National Natural Science Foundation of China under Grant No. 11505301 and the Special Program for Applied Research on Super Computation of the NSFC-Guangdong Joint Fund (the second phase) under Grant No.U1501501. YBZ appreciates valuable discussions with Steven Wong and Amir Khan. We would like to thank the MOMENT working group for fruitful discussions concerned with neutrino fluxes.

\section{References}

[1] B. Aharmim et al. Combined Analysis of all Three Phases of Solar Neutrino Data from the Sudbury Neutrino Observatory. Phys. Rev., C88:025501, 2013.

[2] R. Wendell et al. Atmospheric neutrino oscillation analysis with sub-leading effects in Super-Kamiokande I, II, and III. Phys. Rev., D81:092004, 2010.

[3] S. Abe et al. Precision Measurement of Neutrino Oscillation Parameters with KamLAND. Phys. Rev. Lett., 100:221803, 2008.

[4] K. Abe et al. Precise Measurement of the Neutrino Mixing Parameter $\theta_{23}$ from Muon Neutrino Disappearance in an Off-Axis Beam. Phys. Rev. Lett., 112(18):181801, 2014.

[5] P. Adamson et al. First measurement of muon-neutrino disappearance in NOvA. Phys. Rev., D93(5):051104, 2016.

[6] Y. Abe et al. Improved measurements of the neutrino mixing angle $\theta_{13}$ with the Double Chooz detector. JHEP, 10:086, 2014. [Erratum: JHEP02,074(2015)].

[7] Feng Peng An et al. Measurement of electron antineutrino oscillation based on 1230 days of operation of the Daya Bay experiment. Phys. Rev., D95(7):072006, 2017.

[8] J. H. Choi et al. Observation of Energy and Baseline Dependent Reactor Antineutrino Disappearance in the RENO Experiment. Phys. Rev. Lett., 116(21):211801, 2016.

[9] G. L. Fogli, E. Lisi, A. Marrone, D. Montanino, A. Palazzo, and A. M. Rotunno. Global analysis of neutrino masses, mixings and phases: entering the era of leptonic CP violation searches. Phys. Rev., D86:013012, 2012.

[10] D. V. Forero, M. Tortola, and J. W. F. Valle. Neutrino oscillations refitted. Phys. Rev., D90(9):093006, 2014.

[11] M. C. Gonzalez-Garcia, Michele Maltoni, and Thomas Schwetz. Updated fit to three neutrino mixing: status of leptonic CP violation. JHEP, 11:052, 2014.

[12] Ivan Esteban, M. C. Gonzalez-Garcia, Michele Maltoni, Ivan Martinez-Soler, and Thomas Schwetz. Updated fit to three neutrino mixing: exploring the accelerator-reactor complementarity. JHEP, 01:087, 2017.

[13] V. Barger, D. Marfatia, and K. Whisnant. Off-axis beams and detector clusters: Resolving neutrino parameter degeneracies. Phys. Rev., D66:053007, 2002. 
[14] Hisakazu Minakata, Hiroshi Nunokawa, and Stephen J. Parke. Parameter degeneracies in neutrino oscillation measurement of leptonic CP and T violation. Phys. Rev., D66:093012, 2002.

[15] K. Abe et al. Measurements of neutrino oscillation in appearance and disappearance channels by the T2K experiment with $6.6 \times 10^{20}$ protons on target. Phys. Rev., D91(7):072010, 2015.

[16] P. Adamson et al. First measurement of electron neutrino appearance in NOvA. Phys. Rev. Lett., 116(15):151806, 2016.

[17] R. Acciarri et al. Long-Baseline Neutrino Facility (LBNF) and Deep Underground Neutrino Experiment (DUNE). 2015.

[18] K. Abe et al. A Long Baseline Neutrino Oscillation Experiment Using J-PARC Neutrino Beam and Hyper-Kamiokande. 2014.

[19] J. Cao et al. Roadmap for the international, accelerator-based neutrino programme. 2017.

[20] Fengpeng An et al. Neutrino Physics with JUNO. J. Phys., G43(3):030401, 2016.

[21] Soo-Bong Kim. New results from RENO and prospects with RENO-50. Nucl. Part. Phys. Proc., 265-266:93-98, 2015.

[22] Shakeel Ahmed et al. Physics Potential of the ICAL detector at the India-based Neutrino Observatory (INO). Pramana, 88(5):79, 2017.

[23] M. G. Aartsen et al. Letter of Intent: The Precision IceCube Next Generation Upgrade (PINGU). 2014.

[24] S. Adrian-Martinez et al. Letter of intent for KM3NeT 2.0. J. Phys., G43(8):084001, 2016.

[25] Tommy Ohlsson. Status of non-standard neutrino interactions. Rept. Prog. Phys., 76:044201, 2013.

[26] O. G. Miranda and H. Nunokawa. Non standard neutrino interactions: current status and future prospects. New J. Phys., 17(9):095002, 2015.

[27] Jun Cao et al. Muon-decay medium-baseline neutrino beam facility. Phys. Rev. ST Accel. Beams, 17:090101, 2014.

[28] Mattias Blennow, Pilar Coloma, and Enrique FernÃąndez-Martinez. The MOMENT to search for CP violation. JHEP, 03:197, 2016.

[29] Carla Biggio, Mattias Blennow, and Enrique Fernandez-Martinez. General bounds on non-standard neutrino interactions. JHEP, 08:090, 2009.

[30] Joachim Kopp, Manfred Lindner, Toshihiko Ota, and Joe Sato. Non-standard neutrino interactions in reactor and superbeam experiments. Phys. Rev., D77:013007, 2008.

[31] Pouya Bakhti and Yasaman Farzan. CP-Violation and Non-Standard Interactions at the MOMENT. JHEP, 07:109, 2016.

[32] C. Patrignani et al. Review of Particle Physics. Chin. Phys., C40(10):100001, 2016. 